\title{
Nonlinear evolution in the re-summed next-to-leading order of perturbative QCD: Confronting the experimental data
}

\author{
Carlos Contreras, ${ }^{1, *}$ Eugene Levin ${ }^{1,2,3, \dagger}$ and Michael Sanhueza $\oplus^{1, *}$ \\ ${ }^{1}$ Departamento de Física, Universidad Técnica Federico Santa María, \\ Avenida España 1680, Casilla 110-V, Valparaíso, Chile \\ ${ }^{2}$ Centro Científico- Tecnológico de Valparaíso, Avda. España 1680, Casilla 110-V, Valparaíso, Chile \\ ${ }^{3}$ Department of Particle Physics, School of Physics and Astronomy, Raymond and Beverly Sackler \\ Faculty of Exact Science, Tel Aviv University, Tel Aviv, 69978, Israel
}

(Received 27 June 2021; accepted 2 December 2021; published 22 December 2021)

\begin{abstract}
In this paper we compare the experimental HERA data with the next-to-leading order approach (NLO) of [Eur. Phys. J. C 80, 1029 (2020)]. This approach includes the re-summed NLO corrections to the kernel of the evolution equation, the correct asymptotic behavior in the $\mathrm{NLO}$ at $\tau=r^{2} Q_{s}^{2} \gg 1$; the impact parameter dependence of the saturation scale in accord with the Froissarrt theorem as well as the nonlinear corrections. In this paper, we successfully describe the experimental data with the quality, which is not worse, than in the leading order fits with larger number of the phenomenological parameters. It is demonstrated, that the data could be described, taking into account both the diffusion on $\ln \left(k_{T}\right)$, which stems from perturbative QCD, and the Gribov's diffusion in impact parameters. It is shown an ability to describe the data at rather large values of $\bar{\alpha}_{S}$.
\end{abstract}

DOI: $10.1103 /$ PhysRevD.104.116020

\section{INTRODUCTION}

The goal of this paper is to compare with the experimental (HERA) data the next-to-leading order approach (NLO) of Ref. [1]. In Ref. [1], we develop the approach in which we include the re-summation procedure, suggested in Refs. [2-4], to fix the BFKL kernel in the NLO. In particular, we introduce the rapidity variable, which plays the role of the "evolution time," in the same way as in Ref. [5]. However, we suggest a different way to account for the nonlinear corrections, than in Ref. [5], which leads to additional change of the NLO kernel of the evolution equation. The advantage of our kernel of the BFKL equation [6,7], is that the scattering amplitude satisfies the high energy limits, which follows from the approach of Ref. [8] (see Refs. [9,10]) to the NLO Balitsky-Kovchegov (BK) [11] evolution [12-18].

We firmly believe that finding the correct NLO approximation for the nonlinear evolution is one of the most important and urgent problem in the theoretical description

\footnotetext{
*arlos.contreras@usm.cl

†leving@tauex.tau.ac.il, eugeny.levin@usm.cl

”michael.sanhueza.roa@gmail.com
}

Published by the American Physical Society under the terms of the Creative Commons Attribution 4.0 International license. Further distribution of this work must maintain attribution to the author(s) and the published article's title, journal citation, and DOI. Funded by SCOAP. of the high energy scattering. Indeed, in the color glass condensate (CGC) approach, which is the only candidate for an effective theory at high energies (see Ref. [19] for a review), the two essential parameters, that determine the high energy scattering, calculated in leading order of perturbative QCD [6,11,20-28] turns out to be in an apparent contradiction with the experimental data. The first one is the BFKL Pomeron [6] intercept, which is equal to $2.8 \bar{\alpha}_{S}$ and leads to the energy behavior of the scattering amplitude $N \propto \exp \left(2.8 \bar{\alpha}_{S} \ln \left(\frac{1}{x}\right)\right)$. The second is the energy behavior of the new dimensional scale: saturation momentum $Q_{s}^{2} \propto \exp \left(4.88 \bar{\alpha}_{S} \ln \left(\frac{1}{x}\right)\right)$. Both show the increase in the leading order CGC approach, which cannot be reconciled with the available experimental data. So, the large NLO corrections appear as the only way out, now as well as two decades ago. The needs for the NLO corrections for describing the experimental data has long been realized and many attempts to include these corrections have been made (see, for example, Refs. [5,9,29-34]). In this paper we wish to describe the HERA data using approach of Ref. [1].

In the next section we will outline the main results of Ref. [1] and will specify our theoretical description of the dipole scattering amplitude. However, the current stage of our theoretical understanding of nonperturbative QCD is such, that we have to build a model. We need to take into account the nonperturbative corrections that will reproduce the correct, exponentially decreasing at large impact parameters $(b)$ scattering amplitude. It has been 
demonstrated in Refs. [35-38], that the CGC equations [11,23-28] as well as all other approaches, based on perturbative QCD, lead to the amplitude that increases as a power of energy, resulting in the violation of the Froissart theorem [39]. ${ }^{1}$ Unfortunately, without a theoretical control on nonperturbative QCD we have to use a phenomenological approach to model the large $b$ behavior. In this paper we will exploit two approaches:

(1) the nonperturbative behavior of the saturation scale, which we will parametrize as follows:

$$
Q_{s}^{2}(b, Y) \propto(S(b, m))^{\frac{1}{\bar{r}}}
$$

where $S(b)$ is the Fourier image of $S\left(Q_{T}\right)=$ $1 /\left(1+\frac{Q_{T}^{2}}{m^{2}}\right)^{2}$ and the value of $\bar{\gamma}$ we will discuss below. In the vicinity of the saturation scale such $b$ dependance results in the large $b$-dependence of the scattering amplitude, which is proportional to $\exp (-m b)$ at $b \gg 1 / m$, in accordance of the Froissart theorem [39]. In addition, we reproduce the large $Q_{T}$ dependence of this amplitude proportional to $Q_{T}^{-4}$ which follows from the perturbative QCD calculation [45]. Theoretically the fact that we can absorb the nonperturbative $b$-dependence in $Q_{s}(Y, b)$ (see Eq. (1) for example), follows from the semiclassical approach to BK equation [46] and has been widely used in all, so called, saturation models $[9,47-66]$.

(2) in Refs. [67-76] it is made an attempt to incorporate in the BFKL equation the Gribov's diffusion [77] in impact parameter $(b)$. As the result of this, the following formula for the saturation scale was suggested [74,76]:

$$
Q_{s}^{2}(b, Y) \propto \exp \left(-\frac{3}{4} \mathcal{Z}\right) \text { with } \mathcal{Z}=\left(\frac{b^{4}}{4 \alpha_{\mathrm{eff}}^{\prime 2} Y}\right)^{1 / 3}
$$

where $\alpha_{\text {eff }}^{\prime 2}$ is a new dimensional nonperturbative parameter which controls the Gribov's diffusion.

The paper is organized as follows. In Sec. II we will give a brief review of the approach that we have developed in Ref. [1]. Next we will discuss the procedure of obtaining the NLO kernel of the BFKL equation based on the anomalous dimensions for re-summed NLO corrections, which is suggested in Refs. [2-4,78]. Then we will consider the modification that we need to introduce in the NLO kernel to account for correct behavior of the scattering amplitude at high energy and the structure of the nonlinear equation.

\footnotetext{
${ }^{1}$ It should be noted that NLO corrections to the kernel of CGC evolution equations do not change the powerlike behavior of the scattering amplitude but improve the situation moving the violation of the Froissart theorem to larger values of $b$ [40-44].
}

In Sec. III we specify our approach, which we use for describing the experimental data. In particular, we introduce the phenomenological parameters, which have to be calculated in the nonperturbative QCD approach, and discuss their physical meaning and the range of possible values. In Sec. IV we collect the results of the fit of the experimental data on DIS. We summarize our results in the conclusion.

\section{LEADING TWIST APPROXIMATION FOR NONLINEAR EVOLUTION IN THE NLO- A RECAP}

\section{A. Re-summed anomalous dimensions in NLO and the kernel of linear evolution}

The general solution to the linear equation has the following form:

$$
\begin{aligned}
N(Y, \boldsymbol{r}, \boldsymbol{b}) & =\int_{\epsilon-i \infty}^{\epsilon+i \infty} \frac{d \gamma}{2 \pi i} e^{\omega(\gamma) Y} \phi_{\gamma}(\boldsymbol{r}, \boldsymbol{b}, \boldsymbol{R}) \phi_{\mathrm{in}}(\gamma, R) \\
& =\int_{\epsilon-i \infty}^{\epsilon+i \infty} \frac{d \omega}{2 \pi i} e^{\omega Y} \phi_{\gamma(\omega)}(\boldsymbol{r}, \boldsymbol{b}, \boldsymbol{R}) \phi_{\mathrm{in}}(\omega, R)
\end{aligned}
$$

where $N$ is the scattering amplitude of the dipole with size $r$ at the impact parameter $b . Y$ is the rapidity of the dipole. $\phi_{\gamma}(\boldsymbol{r}, \boldsymbol{b})$ is the eigenfunction of the BFKL equation which has the general form:

$\phi_{\gamma}(\boldsymbol{r}, \boldsymbol{R}, \boldsymbol{b})=\left(\frac{r^{2} R^{2}}{\left(\boldsymbol{b}+\frac{1}{2}(\boldsymbol{r}-\boldsymbol{R})\right)^{2}\left(\boldsymbol{b}-\frac{1}{2}(\boldsymbol{r}-\boldsymbol{R})\right)^{2}}\right)^{\gamma}=e^{\gamma \xi}$

where $R$ is the size of the target. $\phi_{\text {in }}$ can be found from the initial condition at $Y=0$.

The eigenvalues $\omega(\gamma)$ in the NLO has been calculated in Refs. $[79,80]$. However, it turns out that these eigenvalues have singularities at $\gamma \rightarrow 1$ which has been re-summed taking into account the high order corrections in Refs. [2-4,78]. Finally, $\omega_{\mathrm{NLO}}\left(\bar{\alpha}_{S}, \gamma\right)$ has the form [2-4]:

$\omega_{\mathrm{NLO}}\left(\bar{\alpha}_{S}, \gamma\right)=\bar{\alpha}_{S}\left(\chi_{0}\left(\omega_{\mathrm{NLO}}, \gamma\right)+\omega_{\mathrm{NLO}} \frac{\chi_{1}\left(\omega_{\mathrm{NLO}}, \gamma\right)}{\chi_{0}\left(\omega_{\mathrm{NLO}}, \gamma\right)}\right)$

The analytical expressions for functions $\chi_{0}\left(\omega_{\mathrm{NLO}}, \gamma\right)$ and $\chi_{1}\left(\omega_{\mathrm{NLO}}, \gamma\right)$ can be found in Refs. [1-4]. Equation (5) for $\gamma \rightarrow 1$ has the form:

$$
\omega=\frac{\bar{\alpha}_{S}}{1-\gamma+\omega}
$$

which leads to

$$
\omega(\gamma)=\frac{1}{2}\left(-(1-\gamma)+\sqrt{4 \bar{\alpha}_{S}+(1-\gamma)^{2}}\right) .
$$

As it is shown in Ref. [1] Eq. (7) corresponds to the kernel of Ref. [5]. Resolving Eq. (6) with respect to $\gamma[1,81]$ we obtain: 


$$
1-\gamma=\frac{\bar{\alpha}_{S}}{\omega}-\omega,
$$

Eq. (8) gives the simple equation

$$
\begin{aligned}
\frac{\partial}{\partial \eta} \tilde{N}\left(\xi^{\prime}, \eta ; b\right) & =\bar{\alpha}_{S} \int^{\xi^{\prime}} d \xi^{\prime \prime} \tilde{N}\left(\xi^{\prime \prime}, \eta ; b\right) ; \\
\frac{\partial^{2}}{\partial \eta \partial \xi^{\prime}} \tilde{N}\left(\xi^{\prime}, \eta ; b\right) & =\bar{\alpha}_{S} \tilde{N}\left(\xi^{\prime}, \eta ; b\right),
\end{aligned}
$$

for the amplitude $\tilde{N}\left(\xi^{\prime}, \eta ; b\right)=N\left(\xi^{\prime}, \eta ; b\right) / r^{2} . \quad \eta=Y-\xi^{\prime}$ is a new energy variable, which corresponds correct time ordering in double log approximation (DLA) [5]. In Eq. (8) $\xi^{\prime}=-\xi$.

\section{B. Nonlinear equation and the feedback to the kernel of the linear evolution}

The general structure of the nonlinear BalitskyKovchegov equation [11] has the following form:

$$
\begin{aligned}
\frac{\partial}{\partial Y} N\left(\boldsymbol{x}_{10}, \boldsymbol{b}, Y ; R\right)= & \bar{\alpha}_{S} \int \frac{d^{2} \boldsymbol{x}_{2}}{2 \pi} K\left(\boldsymbol{x}_{02}, \boldsymbol{x}_{12} ; \boldsymbol{x}_{10}\right)\left(N\left(\boldsymbol{x}_{12}, \boldsymbol{b}-\frac{1}{2} \boldsymbol{x}_{20}, Y ; R\right)+N\left(\boldsymbol{x}_{20}, \boldsymbol{b}-\frac{1}{2} \boldsymbol{x}_{12}, Y ; R\right)-N\left(\boldsymbol{x}_{10}, \boldsymbol{b}, Y ; R\right)\right. \\
& \left.-N\left(\boldsymbol{x}_{12}, \boldsymbol{b}-\frac{1}{2} \boldsymbol{x}_{20}, Y ; R\right) N\left(\boldsymbol{x}_{20}, \boldsymbol{b}-\frac{1}{2} \boldsymbol{x}_{12}, Y ; R\right)\right)
\end{aligned}
$$

where $\boldsymbol{x}_{i k}=\boldsymbol{x}_{i}-\boldsymbol{x}_{k}$ and $\boldsymbol{x}_{10} \equiv \boldsymbol{r}, \boldsymbol{x}_{20} \equiv \boldsymbol{r}^{\prime}$ and $\boldsymbol{x}_{12} \equiv \boldsymbol{r}-\boldsymbol{r}^{\prime}$. $Y$ is the rapidity of the scattering dipole and $\boldsymbol{b}$ is the impact factor. $K\left(\boldsymbol{x}_{02}, \boldsymbol{x}_{12} ; \boldsymbol{x}_{10}\right)$ is the kernel of the BFKL equation. In our approach we wish to preserve this form, but include the NLO corrections to the kernel. In particular, we would like to include the Reggeization term in Eq. (10), which contribute to the linear equation, but has been neglected in DLA [see Eq. (9)]. It should be stressed that only keeping this term we can provide the correct asymptotic behavior at large $Y: N \rightarrow 1$. In Ref. [1] it is shown that the linear equation, which corresponds to Eq. (8) can be rewritten in the form:

$$
\frac{\partial^{2}}{\partial \eta \partial \xi^{\prime}} \tilde{N}\left(\xi^{\prime}, \eta\right)=\bar{\alpha}_{S} \tilde{N}\left(\xi^{\prime}, \eta\right)-\frac{1}{2} \bar{\alpha}_{S} \frac{\partial}{\partial \xi^{\prime}} \tilde{N}\left(\xi^{\prime}, \eta\right)
$$

For the dipole amplitude, $N\left(\xi^{\prime}, Y\right)$, Eq. (8) takes the following form:

$$
\begin{aligned}
\frac{\partial^{2}}{\partial Y \partial \xi^{\prime}} N\left(\xi^{\prime}, Y\right)+\frac{\partial}{\partial Y} N\left(\xi^{\prime}, Y\right)= & \frac{1}{2} \bar{\alpha}_{S} N\left(\xi^{\prime}, Y\right) \\
& -\frac{1}{2} \bar{\alpha}_{S} \frac{\partial}{\partial \xi^{\prime}} N\left(\xi^{\prime}, Y\right)
\end{aligned}
$$

which leads to the eigenvalue $\omega(\gamma)$ :

$$
\omega(\gamma)=\frac{1}{2} \bar{\alpha}_{S} \frac{1+\gamma}{1-\gamma} .
$$

In Ref. [78] the general equation for $\omega$ has been rewritten in the simple form, that takes into account the energy conservation and coincides with Eq. (5) within $7 \%$. In Ref. [1] it is found that this equation leads to a more general form of $\omega(\gamma)$ in the vicinity of $\gamma \rightarrow 1$ :

$$
\omega(\gamma)=\frac{1}{2} \bar{\alpha}_{S} \frac{1+\gamma}{1+\bar{\alpha}_{S}-\gamma}
$$

Using the general equation to determine the critical anomalous dimension and the energy behavior of the saturation scale (see review [19] and Fig. 1):

$\lambda_{\eta}=\frac{\omega\left(\bar{\gamma}_{\eta}\right)}{\bar{\gamma}_{\eta}}=-\frac{d \omega\left(\bar{\gamma}_{\eta}\right)}{d \bar{\gamma}_{\eta}} ; \quad \ln \left(\frac{Q_{s}^{2}(Y)}{Q_{s}^{2}(Y=0)}\right)=\lambda_{\eta} \eta$

we obtain:

$\bar{\gamma}_{\eta}=\sqrt{2+\bar{\alpha}_{S}}-1 ; \quad \lambda_{\eta}=\frac{1}{2} \frac{\bar{\alpha}_{S}}{3+2 \bar{\alpha}_{S}-2 \sqrt{2+\bar{\alpha}_{S}}} ;$

Rewriting Eq. (16) for the saturation momentum as a function of $Y$ we obtain

$\ln \left(\frac{Q_{s}^{2}(Y)}{Q_{s}^{2}(Y=0)}\right)=\lambda Y \quad$ with $\quad \lambda=\frac{\lambda_{\eta}}{1+\lambda_{\eta}} \quad$ and

$$
\bar{\gamma}=\bar{\gamma}_{\eta}\left(1+\lambda_{\eta}\right) .
$$

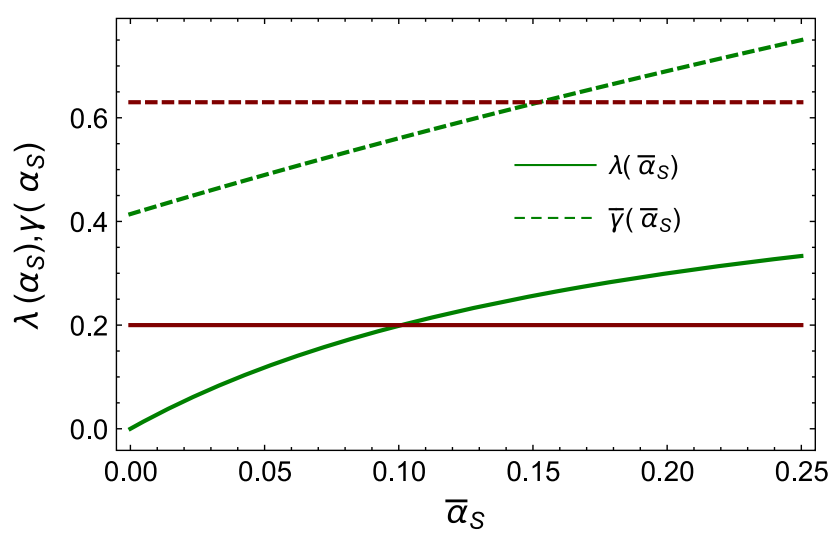

FIG. 1. $\lambda$ and $\bar{\gamma}$ versus $\bar{\alpha}_{S}$. In red we put $\bar{\gamma}=0.63$ which stems from LO estimates and $\lambda=0.2$ which is a typical value that is need to describe DIS data (see for example Ref. [65]). 


\section{NLO BFKL kernel in the saturation domain}

The kernel in $\gamma$-representation: The nonlinear corrections are essential in the region of small values of $x$ (large $Y$ ) where $\tau=r^{2} Q_{s}^{2}>1$. Following Ref. [1] we replace the full kernel of the BFKL equation by the one, which corresponds to the sum of two types of logarithms [8]

$\chi(\gamma)=\left\{\begin{array}{l}\frac{1}{\gamma} \quad \text { for } \tau=r Q_{s}>1 \text { summing }\left(\ln \left(r Q_{s}\right)\right)^{n} ; \\ \frac{1}{1-\gamma} \text { for } \tau=r Q_{s}<1 \text { summing }\left(\ln \left(1 /\left(r \Lambda_{\mathrm{QCD}}\right)\right)\right)^{n}\end{array}\right.$

In the previous section we specified how we changed the kernel in the perturbative QCD region, taking into account he NLO corrections. In the region of large $\tau$ we need to find the anomalous dimension at $\gamma \rightarrow 0$, as it follows from Eq. (18). We found it [1], expanding the anomalous dimension of Ref. [78] in the vicinity of $\gamma \rightarrow 0$. It takes the form:

$$
\omega=\bar{\alpha}_{S}\left\{\frac{1}{\gamma}-\omega\right\}
$$

Resolving Eq. (19) with respect of $\gamma$ we obtain

$$
\gamma=\frac{\bar{\alpha}_{S}}{1+\bar{\alpha}_{S}} \frac{1}{\omega}
$$

which differs from the behavior of the LO kernel, only by replacing $\bar{\alpha}_{S} \rightarrow \bar{\alpha}_{S} /\left(1+\bar{\alpha}_{S}\right)$. Therefore, we can discuss the nonlinear equation in the LO, substituting $\bar{\alpha}_{S} /\left(1+\bar{\alpha}_{S}\right)$ in place of $\bar{\alpha}_{S}$, at the final stage.

The nonlinear equation: In the saturation region where $\tau>1$, the logarithms originate from the decay of a large size dipole, into one small size dipole and one large size dipole [8]. However, the size of the small dipole is still larger than $1 / Q_{s}$. This observation can be translated in the following nonlinear equation (see Ref. [1] for details):

$\frac{\partial^{2} \hat{N}(Y, \boldsymbol{x} ; \boldsymbol{b})}{\partial Y \partial \xi}=\bar{\alpha}_{S}\left\{\left(1-\frac{\partial \hat{N}(Y, \xi ; \boldsymbol{b})}{\partial \xi}\right) \hat{N}(Y, \xi ; \boldsymbol{b})\right\}$

where $\hat{N}(Y, \xi ; \boldsymbol{b})=\int^{\xi} d \xi^{\prime} N\left(Y, \xi^{\prime} ; \boldsymbol{b}\right)$.

For the NLO kernel of Eq. (20), the nonlinear equation has the same form as Eq. (21) but $\bar{\alpha}_{S}$ has to be replaced by $\bar{\alpha}_{S} /\left(1+\bar{\alpha}_{S}\right)$.

The solution: For solving this equation we introduce function $\Omega(Y ; \xi, \boldsymbol{b})$ [8]

$$
N(Y, \xi)=1-\exp (-\Omega(Y, \xi))
$$

Substituting Eq. (22) into Eq. (21) we reduce it to the form

$$
\begin{aligned}
\frac{\partial \Omega(Y, \xi)}{\partial Y} & =\frac{\bar{\alpha}_{S}}{1+\bar{\alpha}_{S}} \tilde{N}(Y, \xi) ; \\
\frac{\partial^{2} \Omega(Y, \xi)}{\partial Y \partial \xi} & =\frac{\bar{\alpha}_{S}}{1+\bar{\alpha}_{S}}(1-\exp (-\Omega(Y, \xi))) \\
\frac{\partial^{2} \Omega\left(\xi_{s} ; \zeta\right)}{\partial \xi_{s} \partial \xi} & =\frac{\partial^{2} \Omega\left(\xi_{s} ; \tilde{\zeta}\right)}{\partial t_{+}^{2}}-\frac{\partial^{2} \Omega\left(\xi_{s} ; \tilde{\zeta}\right)}{\partial t_{-}^{2}} \\
& =\sigma\left(1-\exp \left(-\Omega\left(\xi_{s} ; \zeta\right)\right)\right)
\end{aligned}
$$

where $\lambda$ is given by Eq. (17) and $\sigma=\bar{\alpha}_{S} / \lambda\left(\bar{\alpha}_{S}\right)\left(1+\bar{\alpha}_{S}\right)$. The variables $t_{ \pm}=\xi_{s} \pm \xi$ with $\xi_{s}$ defined as

$$
\xi_{s}=\ln \left(Q_{s}^{2}(Y) / Q_{s}^{2}(Y=0 ; \boldsymbol{b}, \boldsymbol{R})\right)=\lambda Y
$$

The use of this variable indicates the main idea of our approach in the region of $\tau>1$ : we wish to match the solution of the nonlinear Eq. (23b) with the solution of linear equation (see Eq. (12)) in the kinematic region where it has the form [82]:

$$
N=N_{0} \exp (\bar{\gamma} z) \quad \text { with } \quad z=\xi_{s}+\xi
$$

and $\bar{\gamma}$ is determined by Eq. (17). Equation (25) leads to the initial and boundary conditions:

$N\left(r^{2}, Y\right)=N(\tau=1)=N_{0} ;\left.\quad \frac{d \ln \left(N\left(r^{2}, Y\right)\right)}{d \xi}\right|_{\tau=1}=\bar{\gamma}$

Eq. (23b) has a traveling wave solution (see formula 3.4.1.1 of Ref. [83]). For Eq. (23b) in the canonical form the solution takes the form:

$\int_{\Omega_{0}}^{\Omega} \frac{d \Omega^{\prime}}{\sqrt{C_{1}+\frac{2}{\left(\mu^{2}-\kappa^{2}\right)} \sigma\left(\Omega^{\prime}+\exp \left(-\Omega^{\prime}\right)\right)}}=\mu t_{+}+\kappa t_{-}+C_{2}$

where all constants have to be determined from the initial and boundary conditions of Eq. (26) and they take the forms [1]:

$$
C_{2}=0 ; \quad C_{1}=-\frac{2 \sigma}{\mu^{2}}+\Omega_{0}^{2}\left(1-\frac{\sigma}{\mu^{2}}\right) ; \text { and } \mu=\bar{\gamma}
$$

For $\Omega \rightarrow \Omega_{0}$ and if $\Omega_{0} \ll 1$, Eq. (27) can be solved explicitly giving

$\Omega=\Omega_{0}\left\{\cosh \left(\sqrt{\sigma}\left(\xi_{s}+\xi\right)\right)+\frac{\bar{\gamma}}{\sqrt{\sigma}} \sinh \left(\sqrt{\sigma}\left(\xi_{s}+\xi\right)\right)\right\}$ 
Eq. (29) gives the solution which depends only on one variable $z=\xi_{s}+\xi$, and satisfies the initial conditions of Eq. (26).

At large $z$ we obtain the solution [8]:

$\Omega(z)=\frac{\sigma}{2} z^{2}+$ Const; $\quad N(z)=1-$ Const $e^{-\frac{\sigma}{2} z^{2}}$

We wish to stress that Eq. (30) reproduces the asymptotic solution to the BK equation in the NLO, which has been derived in Refs. $[9,10]$, for fixed $\bar{\alpha}_{S}$.

It should be noted that both solutions of Eq. (29) and Eq. (30) can be derived directly from Eq. (23b) assuming $1-\exp (-\Omega) \rightarrow \Omega$ and $1-\exp (-\Omega) \rightarrow 1$ for small $z$ and large $z$, respectively.

\section{OUR APPROACH}

\section{A. Generalities}

The observables in deep inelastic scattering can be expressed through the following scattering amplitudes (see Fig. 2 and Ref. [19] for the review and references therein)

$N(Q, Y ; b)=\int \frac{d^{2} r}{4 \pi} \int_{0}^{1} d z\left|\Psi_{\gamma^{*}}(Q, r, z)\right|^{2} N(r, Y ; b)$

where $Y=\ln \left(1 / x_{B j}\right)$ and $x_{B j}$ is the Bjorken $x . z$ is the fraction of energy carried by quark. $Q$ is the photon virtuality. $b$ denotes the impact parameter of the scattering amplitude.

Equation (31) shows the main features of the interactions at high energies. They go in two stages. The first is the decay of virtual photon in quark-antiquark pair, described by $\left|\Psi_{\gamma^{*}}(Q, r, z)\right|^{2}$ in Eq. (31). For large $Q^{2}\left(Q^{2} \geq Q_{0}^{2}\right.$ with $Q_{0}^{2} \approx 0.7 \mathrm{GeV}^{2}$, see Ref. [84]) the wave function is well known (see Ref. [19] and references therein)

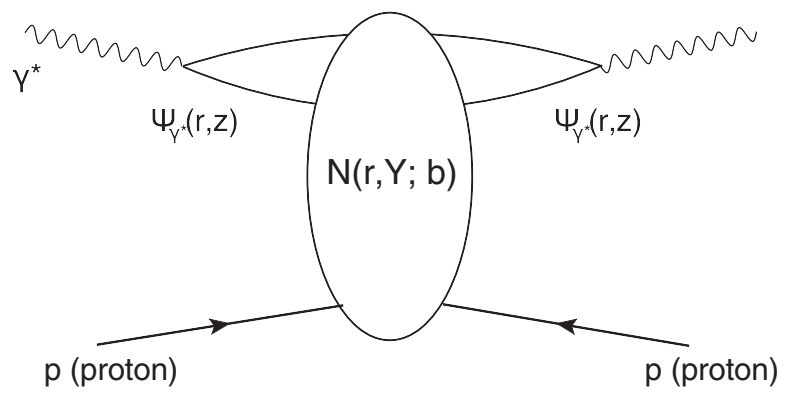

FIG. 2. The graphic representation of Eq. (31) for the scattering amplitude. $Y=\ln \left(1 / x_{B j}\right)$ and $r$ is the size of the interacting dipole. $z$ denotes the fraction of energy that is carried by one quark. $b$ denotes the impact parameter of the scattering amplitude.

$$
\begin{aligned}
(\Psi * \Psi)_{T}^{\gamma^{*}}= & \frac{2 N_{c}}{\pi} \alpha_{\mathrm{em}} \sum_{f} e_{f}^{2}\left\{\left[z^{2}+(1-z)^{2}\right] \epsilon^{2} K_{1}^{2}(\epsilon r)\right. \\
& \left.+m_{f}^{2} K_{0}^{2}(\epsilon r)\right\}, \\
(\Psi * \Psi)_{L}^{\gamma^{*}}= & \frac{8 N_{c}}{\pi} \alpha_{\mathrm{em}} \sum_{f} e_{f}^{2} Q^{2} z^{2}(1-z)^{2} K_{0}^{2}(\epsilon r),
\end{aligned}
$$

where $\mathrm{T}(\mathrm{L})$ denotes the polarization of the photon and $f$ is the flavors of the quarks. $\epsilon^{2}=m_{f}^{2}+Q^{2} z(1-z)$. However, even for DIS the nonperturbative corrections become essential and we cannot use Eq. (32) and Eq. (33) for $Q^{2} \leq Q_{0}^{2}$.

The second is the amplitude $N(r, Y ; b)$ of the interaction of the dipole with the target. We have discussed this amplitude in the previous section and will specify the way how we will use the finding of this section for the practical application to DIS below.

Using Eq. (31), Eq. (32) and Eq. (33) we can write the main observables in DIS:

$$
\begin{aligned}
\sigma_{T, L} & =2 \int d^{2} b N_{T, L}(Q, Y, ; b) ; \\
F_{2}(Q, Y) & =\frac{Q^{2}}{4 \pi^{2} \alpha_{\text {e.m. }}}\left\{\sigma_{T}+\sigma_{L}\right\} ; \\
F_{2}^{c c}(Q, Y) & =\frac{Q^{2}}{4 \pi^{2} \alpha_{\text {e.m. }}}\left\{\sigma_{T}^{c c}+\sigma_{L}^{c c}\right\} ; \\
F_{L}(Q, Y) & =\frac{Q^{2}}{4 \pi^{2} \alpha_{\text {e.m. }}} \sigma_{L} ;
\end{aligned}
$$

$\sigma^{c c}$ in Eq. (34c) are calculated, using Eq. (34a) with $N_{T, L}(Q, Y, ; b)$, which is calculated using Eq. (32) and Eq. (33) for $c$-quarks.

\section{B. The dipole scattering amplitudes}

Based on the approach, briefly discussed in the previous section, we can see three different kinematic regions where we are going to use the scattering amplitude $N\left(Y, \xi^{\prime} ; \boldsymbol{b}\right)$ in three different forms.

(1) For $Q_{s}^{2} r^{2}<1$ (perturbative QCD region) we suggest to use the linear evolution equation of Eq. (12), which has been discussed above. Recalling that in the derivation of this equation we use the DLA in which both $\eta=Y-\xi^{\prime}$ and $\xi^{\prime}$ are considered to be large: $\bar{\alpha}_{S} \eta \xi \gg 1$. For $\eta>0(Y>\xi)$ we can use the experimental data as the initial condition for Eq. (12). The value of the saturation momentum is given by Eq. (17). Since its value turns out to be much less than $Q_{\max }$, which stems from the condition $\xi_{\max }=\ln \left(Q_{\max }^{2} / Q_{0}^{2}\right)=\eta$, we can use the 
DGLAP evolution equation in the next-to-leading order for $Q^{2}>Q_{\max }^{2}$.

(2) For $Q_{s}^{2} r^{2} \sim 1$ (vicinity of the saturation scale) we use the scattering amplitude in the form [82]

$$
N(r, \eta ; b)=N_{0} \quad\left(Q_{s}^{2}(Y, b) r^{2}\right)^{\bar{\gamma}}
$$

with $\bar{\gamma}$ from Eq. (17).

(3) For $Q_{s}^{2} r^{2} \gg 1$ (saturation region), we propose to use the solution to the nonlinear equation of Eq. (27).

For $\tau<1$ we have to solve the linear equation with some initial condition which we need to take from the experimental data. This solution will give us the scattering amplitude in the vicinity of the saturation scale where the scattering amplitude can be estimated using Eq. (35). We use a different way to introduce the parameters from the experimental data: we expand Eq. (35) to the region $\tau<1$, replacing $\bar{\gamma}$ by following expression:

$$
\bar{\gamma} \rightarrow \bar{\gamma}+\frac{\ln (1 / \tau)}{\kappa \lambda Y} ; \quad \text { with } \quad \kappa=\frac{\frac{d^{2} \omega\left(\bar{\gamma}_{\eta}\right)}{d \bar{\gamma}_{\eta}^{2}}}{\frac{d \omega\left(\bar{\gamma}_{\eta}\right)}{d \bar{\gamma}_{\eta}}} .
$$

Equation (36) is derived in Ref. [85] and the experience with the saturation models [9,47-66] shows that it is described the experimental data for $x \leq 0.1$ quite well. Hence, in our approach the values of all phenomenological parameters of Eq. (35) (see below) should be determined from the experimental data.

The mass of the $c$-quark (about $m_{c}=1.4 \mathrm{GeV}$ ) is not small and we took this into account replacing $x$ in the $c \bar{c}$ scattering by $x^{c}=\left(1+\frac{4 m_{c}^{2}}{Q^{2}}\right) x$.

For $\tau=Q_{s}^{2} r^{2} \gg 1$ we need to use the solution of Eq. (27) to the nonlinear evolution equation. However, it has been found in Ref. [86] that the following formula:

$N(z)=a(1-\exp (-\Omega(z)))+(1-a) \frac{\Omega(z)}{1+\Omega(z)}$

with $\Omega(z)$ from Eq. (29) and with $a=0.65$ describes the exact solution within accuracy less that $2.5 \%$. Therefore, we use Eq. (37) in our attempts to describe the HERA data.

\section{Phenomenological input}

As has been mentioned above, we need to set the initial conditions at $Y=0$ for the linear evolution equation for $\tau \leq 1$. In this section we wish to clarify how we introduce the phenomenological parameters to describe these conditions. The first one we have considered: $N_{0}$ which determines the scattering amplitude at $\tau=1$. Two other parameters describe the saturation momentum at $Y=0$ : the value of $Q_{s}$ at $b=0$ and the behavior as a function of $b$. collecting everything that we have discussed about $Q_{s}$ we use two expression for the saturation momentum:
1. $Q_{s}^{(1) 2}(Y, b)=Q_{s}^{(1) 2}(Y=0, b=0) e^{-m b} e^{\lambda Y}=Q_{0}^{2} e^{-m b} e^{\lambda Y}$;

2. $Q_{s}^{(2) 2}(Y, b)=Q_{s}^{(2) 2}(Y=0, b=0) e^{-\frac{3}{4} \mathcal{Z}} e^{\lambda Y}=Q_{0}^{2} e^{\lambda Y} e^{-\frac{3}{4} \mathcal{Z}}$;

where $\mathcal{Z}$ and $\lambda$ are defined in Eq. (2) and Eq. (17), respectively. The values of these parameters have to be found from fitting of the experimental data. The experience with such fitting gives $Q_{0}^{(1) 2}=Q_{0}^{(2) 2}=0.15-0.25 \mathrm{GeV}^{2}$. $m$ we can estimate, assuming that $\left\langle b^{2}\right\rangle=6 / m^{2}=R^{2}$ where $R$ is the electromagnetic radius of the proton. Hence we expect $m \approx 0.55 \mathrm{GeV}$ but we have to remember that $m$ characterizes the distributions of the gluons in the proton which can be quite different from the quarks. ${ }^{2} \alpha_{\text {eff }}^{\prime 2}$ in $\mathcal{Z}$ has been evaluated in Ref. [74] with $\alpha_{\text {eff }}^{\prime 2} \approx 0.1 \mathrm{GeV}^{-2}$ for $\bar{\alpha}_{S}=0.2$.

Finally, we introduce three phenomenological parameters from the initial conditions whose values have to be found from the fit of the experimental data. The masses of quarks, that determine the wave function of the virtual photon, determine the infrared behavior of the wave function. We take two sets of them: the current masses and the masses of light quarks are equal to $140 \mathrm{MeV}$ which is the typical infrared cutoff in our approach.

\section{RESULTS OF THE FITS}

Using the approach, that has been discussed in the previous section, we attempt to describe the most accurate data for the deep inelastic structure function $F_{2}$ [87]. The implicit parameters of our approach are the restrictions of the kinematic region of the experimental data that we include in the fit. We chose: $0.85 \mathrm{GeV}^{2} \leq Q^{2} \leq 27 \mathrm{GeV}^{2}$ and $x \leq 0.01$. The lower limit of $Q^{2}$ stems from nonperturbative correction to the wave function of the virtual photon, while the upper limit originates from two restrictions: $x \leq 0.01$ and contribution of the additional term in $\bar{\gamma}$ of Eq. (36) is small. The choice of the largest $x$ is dictated by the needs to have low $x$ for legitimate use of our theoretical formulas and the practical wish to use in the fit as more data as possible.

In Table I we show the values of parameters from our fits. It should be noted that we did not fit the mass of the quarks but use two sets of them: the masses of light quarks are equal to $m=140 \mathrm{MeV}$, which we consider as the typical infrared cutoff in our approach (sets 1 and 2); and the current masses (sets 3 and 4). We used the data for the inclusive $F_{2}$ in the kinematic region: $0.85 \mathrm{GeV}^{2} \leq Q^{2} \leq 27 \mathrm{GeV}^{2}$ and

\footnotetext{
${ }^{2}$ Actually, the above estimates should be made for the scattering amplitude $N=N_{0}\left(r^{2} Q_{s}^{2}\right)^{\bar{\gamma}}$, which leads to $m \approx 0.34 \mathrm{GeV}$ : with this estimates we need to compare the value of $m$ from the Table I.
} 
TABLE I. Parameters of the model. $\bar{\alpha}_{S}, N_{0}, m\left(\alpha_{\text {eff }}\right)$ and $Q_{0}^{2}$ are fitted parameters. Two sets of quark masses are chosen: the current masses (sets 3 and 4) and the masses of light quarks are equal to $140 \mathrm{MeV}$ (sets 1 and 2), which is the typical infrared cutoff in our approach. For the sets 2 and 4 the value of $\bar{\alpha}_{S}=0.2$ is fixed and only three parameters were chosen from the fit.

\begin{tabular}{|c|c|c|c|c|c|c|c|c|c|c|}
\hline \multirow[b]{2}{*}{ Set } & \multicolumn{5}{|c|}{ Dipole amplitude } & \multicolumn{4}{|c|}{ Wave function } & \multirow{2}{*}{$\frac{\chi^{2} / \text { d.o.f. }}{0.85 \leq Q^{2} \leq 27 \mathrm{GeV}^{2}}$} \\
\hline & $\bar{\alpha}_{S}$ & $N_{0}$ & $Q_{0}^{2}\left(\mathrm{GeV}^{2}\right)$ & $\mathrm{m}(\mathrm{GeV})$ & $\alpha_{\text {eff }}\left(\mathrm{GeV}^{-2}\right)$ & $m_{u}(\mathrm{MeV})$ & $m_{d}(\mathrm{MeV})$ & $m_{s}(\mathrm{MeV})$ & $m_{c}(\mathrm{GeV})$ & \\
\hline 1 & 0.091 & 0.236 & 0.998 & 0.612 & $\mathrm{n} / \mathrm{a}$ & 140 & 140 & 140 & 1.4 & $124.9 / 133=0.93$ \\
\hline 2 & 0.20 & 0.25 & 1.00 & 0.551 & $\mathrm{n} / \mathrm{a}$ & 140 & 140 & 140 & 1.4 & $61.99 / 66=0.93$ \\
\hline 3 & 0.096 & 0.448 & 0.921 & 0.840 & $\mathrm{n} / \mathrm{a}$ & 2.3 & 4.8 & 95 & 1.4 & $117.2 / 133=0.88$ \\
\hline 4 & 0.20 & 0.343 & 0.999 & 1.300 & $\mathrm{n} / \mathrm{a}$ & 2.3 & 4.8 & 95 & 1.4 & $91.74 / 66=1.39$ \\
\hline 5 & 0.038 & 0.599 & 1.284 & $\mathrm{n} / \mathrm{a}$ & 0.216 & 140 & 140 & 140 & 1.4 & $175 / 133=1.31$ \\
\hline 6 & 0.043 & 0.565 & 1.429 & $\mathrm{n} / \mathrm{a}$ & 0.149 & 2.3 & 4.8 & 95 & 1.4 & $143.7 / 133=1.08$ \\
\hline
\end{tabular}

$x \leq 0.01$, for finding the parameters while $F_{2}^{c c}$ as well as $F_{2}$ outside of this kinematic region (for small values of $Q^{2} \leq$ $0.85 \mathrm{GeV}^{2}$ or $Q^{2} \geq 27 \mathrm{GeV}^{2}$ ), are compared with the experimental data, to demonstrate our ability to describe the experimental data. The quality of the fit one can see from Fig. 3. In Fig. 4 we show the data on $F_{2}$ as function of $Q^{2}$ at different values of $x$. This figure illustrates that we can describe the data for $x \leq 0.013$. In addition, we show in Fig. $3 F_{2}$ for $27 \leq Q^{2} \leq 60 \mathrm{GeV}^{2}$. The quality of the fit is not very good due to the small experimental errors. Indeed, $\chi^{2} /$ d.o.f. for the range of $Q^{2}$ from $0.85 \mathrm{GeV}^{2}$ to $60 \mathrm{GeV}^{2}$ is equal to 2.16 (set 1 ), 1.93 (set 3) and 3.19 (set 5). The reason for this stems from the approximate character of Eq. (36). The contributions of the nonlinear corrections are negligible for such large photon virtualities and, therefore, our approach coincides with the one of Ref. [5] (see also Ref. [32]), which describes the data very well. We believe, that we demonstrated that our approach can describe the experimental data at rather small values of $Q^{2}$ where the nonlinear corrections give an essential contribution. For large $Q^{2}$ our approach coincides with the one of Ref. [5] which takes into account the linear evolution in much better way than we [32].

Figure 5 demonstrates how our fits describe the experimental data on $F_{2}^{c c}$ while Fig. 6 shows that our fit is able to reproduce the data at low values of $Q^{2}$. One can see that the agreement with the experimental data is good even at low $Q^{2}$. It should be stressed that two sets of the light quark mass give the same description illustrating a possibility to use the wave function of the virtual photon in perturbative QCD at rather low values of $Q^{2}$.
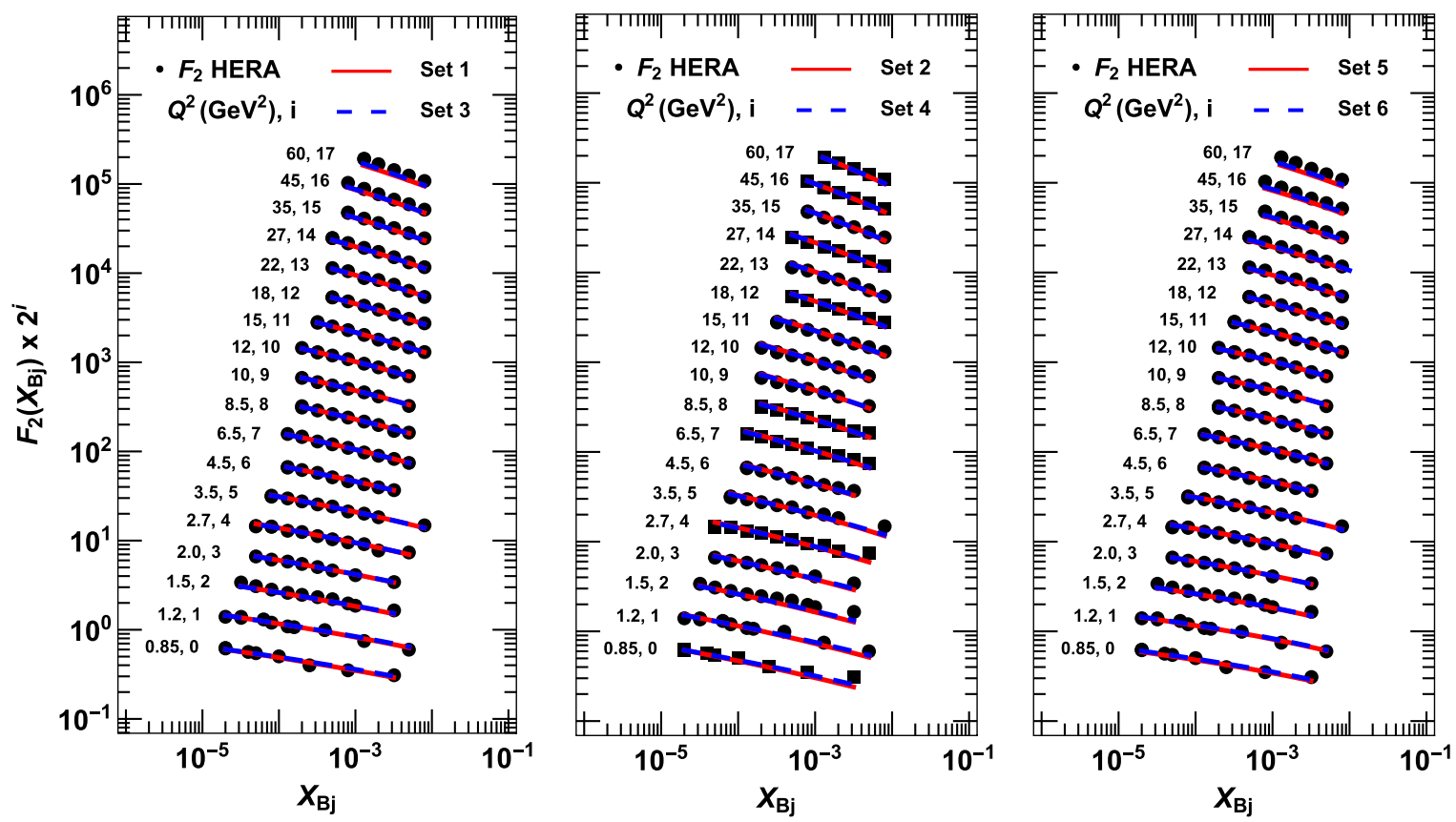

FIG. 3. Comparison with the experimental data of Refs. [87,88] of the sets shown in table I. The experimental data as well as the theoretical estimates are multiplied by factor $2^{i}$ and the values of $i$ are specified in the figures. In the panel with sets 2 and 4 we use black squares to mark the experimental data that have been used in the fit. 

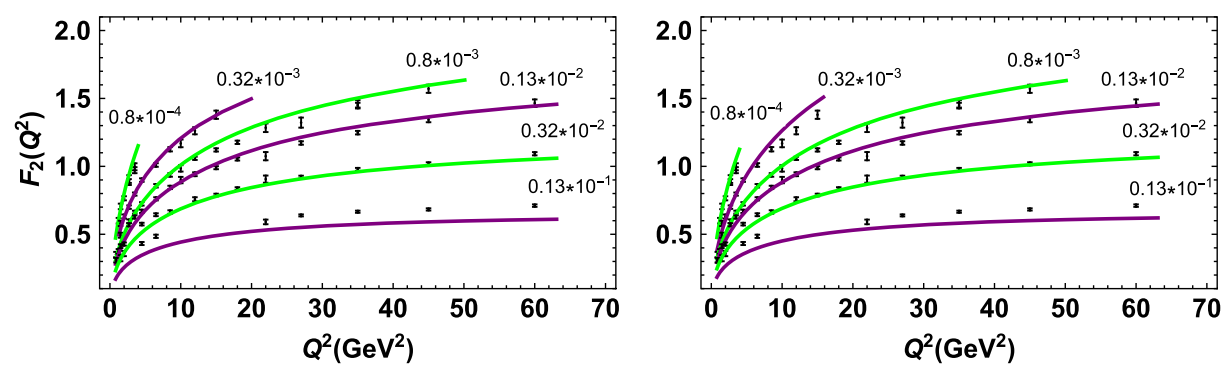

FIG. 4. $\quad F_{2}$ versus $Q^{2}$ at fixed $x$ for the set 2 (left panel) and set 4 (right panel).
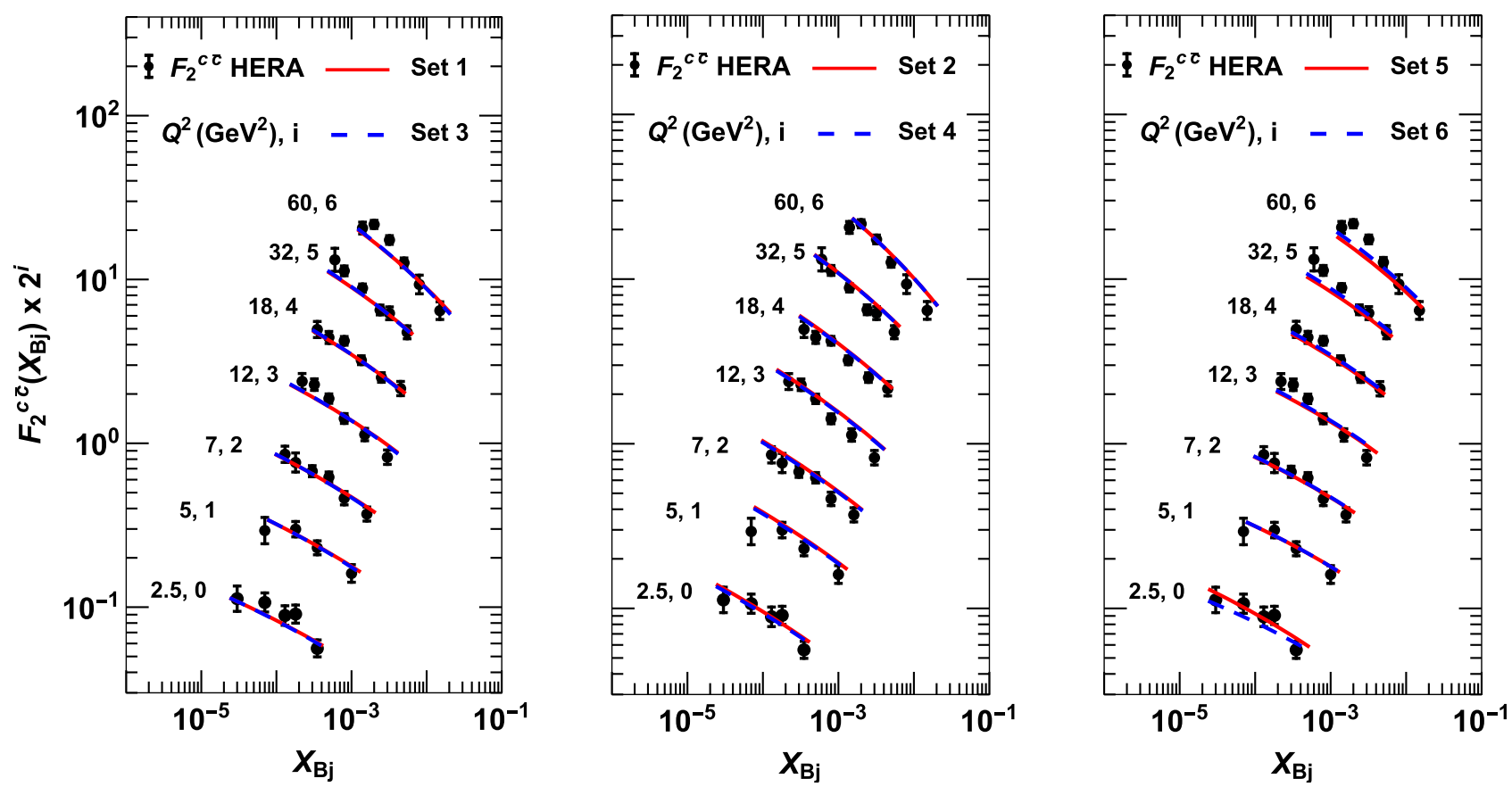

FIG. 5. Comparison our fits with the experimental data on $F_{2}^{c c}$ (see Refs. [87,88]) of the sets shown in the table I. The experimental data as well as the theoretical estimates are multiplied by factor $2^{i}$ and the values of $i$ are specified in the figures.
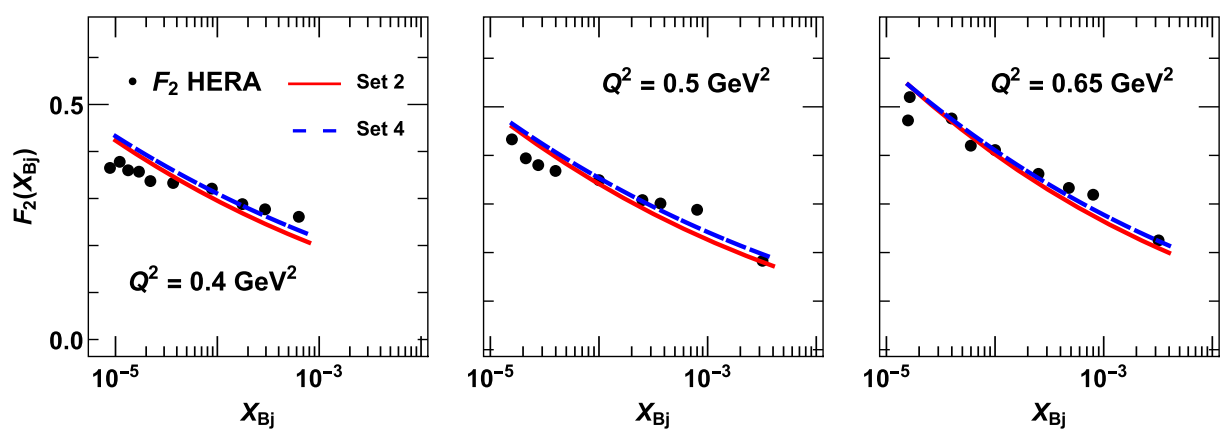

FIG. 6. $\quad F_{2}$ versus $x$ at low $Q^{2}$. Red line for set 2 while the blue one for set 4 .

In addition to $F_{2}$ and $F_{2}^{C C}$ we compare our approach with the experimental data on $F_{L}[89,90]$. In spite of the fact that the data have sufficiently large errors one can see from Fig. 7 that we are able to describe the data quite well.
Sets 2 and 4 need special comments. In these sets we choose $\bar{\alpha}_{S}=0.2$ to illustrate the ability of our approach to overcome the difficulties of next-to-leading approaches in describing the experimental data: the small value of needed 

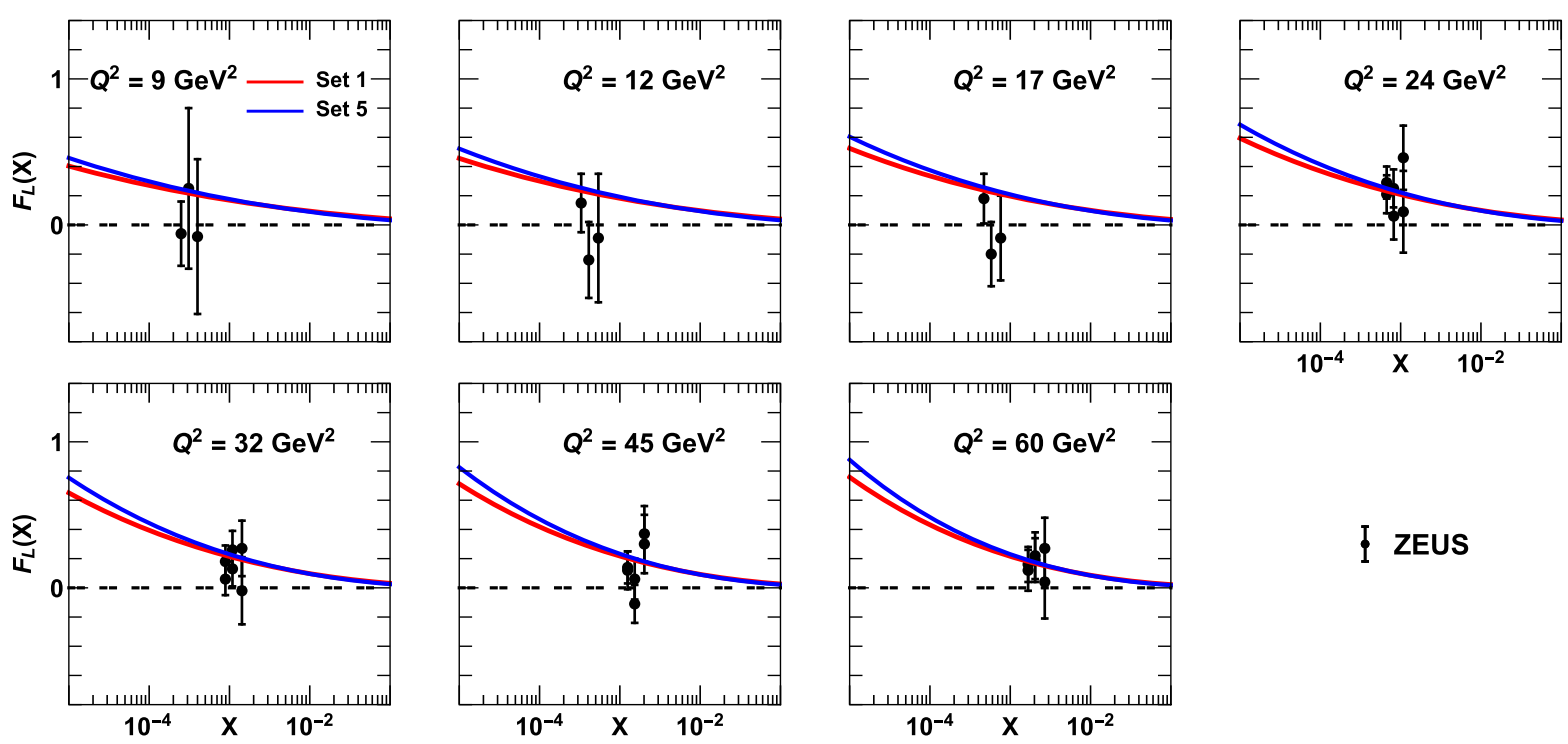

IEUS

FIG. 7. Comparison our fits with the experimental data on $F_{L}$ (see Ref. [90]) of the sets 1 and 5. All other sets lead to the curves which are very close to these two. It is shown the data, extracted from the unconstrained fit (see Ref. [90] for explanation). Note that the data for constrained fit give $F_{L} \geq 0$. All points with negative values of $F_{L}$ in the constrained fits coincide with 0 .

$\bar{\alpha}_{S}$. One can see, that we obtain a good $\chi^{2} /$ d.o.f. but for the specific set of data in the wide kinematic region $0.85 \leq$ $Q^{2} \leq 60 \mathrm{GeV}^{2}$ but choosing a restricted selection of data. Note that these data are marked by the squares in the panel of Fig. 3 with sets 2 and 4.

The general characteristics of our fits are: (1) the possibility to describe the data at rather small values of $Q^{2}$, where the nonlinear corrections turn out to be essential, (2) the large value of $Q_{0}^{2}=1 \mathrm{GeV}^{2}$; (3) rather large value of $m$ in comparison with the estimates from the electromagnetic radius of the proton (see above in footnote 2); (4) the possibility to fit the experimental data, taking into account the Gribov's diffusion for impact parameter dependence of the saturation scale (see panel with sets 5 and 6 in Fig. 3); and (5) an ability to describe the data at rather large values of $\bar{\alpha}_{S}$.

Unfortunately, in spite of large numbers of the papers in which the experimental data have been compared with the saturation models $[9,47-66]$ we can confront our approach only with Refs. [9,66] since in all other papers the assumptions were made that contradict the theoretical information. For example, in the most models $Q_{s}^{2}$ is proportional to $\exp \left(-b^{2} / B\right)$ while this behavior disagrees with the Froissart theorem [39]; or/and the behavior for $r^{2} Q_{s}^{2} \gg 1$ contradicts the approach of Ref. [8]. Comparing our parameters with Ref. [9] one can see that the typical value of $\bar{\alpha}_{S} \approx 0.14-0.15$ in Ref. [9] is less than in sets 2 and 4 . The value of $Q_{0}^{2}$ is about in three times larger than in this paper. The value of $m$ in Ref. [9] is the same as in the electromagnetic form factor of proton, while in this paper $m$ is larger, than in the electro-magnetic form factor of proton in agreement with the new experimental information on the behavior of the two gluon form factors $[91,92]$. Taking into account that $\chi^{2} /$ d.o.f. are better in this paper than in Ref. $[9,66]$, we believe that the theoretical approach of Sec. II, give reliable description of the current experimental data on DIS.

We wish to draw your attention to the sets 5 and 6 , which take into account the different dependence of the saturation momentum on $b$ [see Eq. (38b)]. From Fig. 3 and Fig. 5 one can see that we are able to describe the experimental data. Hence we see that the suggested dependence on rapidity of the impact parameter distribution is in accord with the experimental data.

\section{CONCLUSIONS}

In this paper we compare with the experimental data the NLO approach of Ref. [1]. In this approach we include the re-summation procedure, suggested in Refs. [2-5], to fix the BFKL kernel in the NLO, but we treat differently the nonlinear corrections. The advantage of our treatment is that we reproduce the correct asymptotic behavior at large $\tau=r^{2} Q_{s}^{2}$.

Fixing our phenomenological parameters from the fit to the experimental data on $F_{2}$ DIS structure function in the region: $0.85 \leq Q^{2} \leq 27 \mathrm{GeV}^{2}$ and $x \leq 0.013$, we found that can describe these data with very good $\chi^{2} /$ d.o.f. It should be stressed, that for the first time it is demonstrated that the impact parameter dependence of the saturation scale, which is given by Eq. (38b), is an agreement with the data. We believe that this is an interesting result since Eq. (38b) follows from the first attempt to take into account both the diffusion on $\ln \left(k_{T}\right)$, which stems from perturbative QCD, and the Gribov's diffusion in $b$ [76]. 
Using the phenomenological parameters, that we found from the fit of $F_{2}$, we demonstrated that our approach is able to describe the experimental data both on $F_{2}^{c c}$ and on $F_{2}$ at low values of $Q^{2}=0.4-0.7 \mathrm{GeV}^{2}$. We also reproduce the experimental data on $F_{L}$.

It should be pointed out that our description in the NLO is better or of the same quality as the description by LO with more fitting parameters. Two problems, that we faced in describing the data in the NLO approach, have been partly healed: we are able to fit the data with not small value of $\bar{\alpha}_{S}=0.2$ and the value of the saturation momentum at $Y=0$ is about $Q_{0}^{2} \approx 1 \mathrm{GeV}^{2}$ instead of $3 \mathrm{GeV}^{2}$ of Ref. [9]. However, we are aware that $Q_{0}=1 \mathrm{GeV}$ is rather large (see for example Ref. [93]).

Concluding we wish to mentioned that the model that we developed here, is the only one which includes (i) the resummed NLO corrections; (ii) the correct theoretical behavior at large $\tau$; (iii) the large $b$ dependence of the saturation scale in accord with the Froissart theorem as well as the nonlinear evolution. We believe that it will be useful for further discussion of the high energy scattering in QCD.
Our resulting scattering amplitude can be used in other phenomenological applications as for example: production of dijets in $\mathrm{p}-\mathrm{p}$ and $\mathrm{p}-\mathrm{Pb}$ collisions (see Ref. [30]) or the structure of the soft Pomeron in CGC (see Ref. [94])

\section{ACKNOWLEDGMENTS}

We thank our colleagues at Tel Aviv university and UTFSM for encouraging discussions. Our special thanks go to E. Gotsman for all his remarks and suggestions on this paper, and to Yuri Ivanov for technical support of the USM HPC cluster. M. S. thanks M. Arriagada and C. del Valle from Universidad de la Frontera, and S. Nauto and J. Vidal from Universidad de Playa Ancha, as well as M. Siddikov from UTFSM for their valuable advices on programming. This research was supported by ANID PIA/APOYO AFB180002 (Chile), Fondecyt (Chile) Grants No. 1180118 and No. 1191434, Conicyt Becas (Chile) and Grant No. PIIC 009/2021, DPP, Universidad Técnica Federico Santa María.
[1] C. Contreras, E. Levin, R. Meneses, and M. Sanhueza, Nonlinear equation in the re-summed next-to-leading order of perturbative QCD: the leading twist approximation, Eur. Phys. J. C 80, 1029 (2020).

[2] G. P. Salam, A resummation of large subleading corrections at small x, J. High Energy Phys. 07 (1998) 019.

[3] M. Ciafaloni, D. Colferai, and G. P. Salam, Renormalization group improved small $x$ equation, Phys. Rev. D 60, 114036 (1999).

[4] M. Ciafaloni, D. Colferai, G. P. Salam, and A. M. Stasto, Renormalization group improved small $x$ Green's function, Phys. Rev. D 68, 114003 (2003).

[5] B. Ducloe, E. Iancu, A. H. Mueller, G. Soyez, and D. N. Triantafyllopoulos, Non-linear evolution in QCD at highenergy beyond leading order, J. High Energy Phys. 04 (2019) 081 and references therein.

[6] V. S. Fadin, E. A. Kuraev, and L. N. Lipatov, On the Pomeranchuk singularity in asymptotically free theories, Phys. Lett. 60B, 50 (1975); E. A. Kuraev, L. N. Lipatov, and V. S. Fadin, The Pomeranchuk singularity in nonabelian gauge theories, Zh. Eksp. Teor. Fiz. 72, 377 (1977) [Sov. Phys. JETP 45, 199 (1977)]; I. I. Balitsky and L. N. Lipatov, The Pomeranchuk singularity in quantum chromodynamics, Yad. Fiz. 28, 1597 (1978) [Sov. J. Nucl. Phys. 28, 822 (1978)].

[7] L. N. Lipatov, Small x physics in perturbative QCD, Phys. Rep. 286, 131 (1997); The bare Pomeron in quantum chromodynamics, Zh. Eksp. Teor. Fiz. 90, 1536 (1986) [Sov. Phys. JETP 63, 904 (1986)].

[8] E. Levin and K. Tuchin, Solution to the evolution equation for high parton density QCD, Nucl. Phys. B573, 833 (2000); New scaling at high-energy DIS, Nucl. Phys. A691, 779
(2001); Nonlinear evolution and saturation for heavy nuclei in DIS, Nucl. Phys. A693, 787 (2001).

[9] C. Contreras, E. Levin, R. Meneses, and I. Potashnikova, CGC/saturation approach: A new impact-parameter dependent model in the next-to-leading order of perturbative QCD, Phys. Rev. D 94, 114028 (2016).

[10] W. Xiang, Y. Cai, M. Wang, and D. Zhou, Rare fluctuations of the $S$-matrix at NLO in QCD, Phys. Rev. D 99, 096026 (2019).

[11] I. Balitsky, Operator expansion for high-energy scattering, Nucl. Phys. B463, 99 (1996); Factorization and high-energy effective action, Phys. Rev. D 60, 014020 (1999); Y. V. Kovchegov, Small- $x F_{2}$ structure function of a nucleus including multiple Pomeron exchanges, Phys. Rev. D 60, 034008 (1999).

[12] I. Balitsky, Quark contribution to the small-x evolution of color dipole, Phys. Rev. D 75, 014001 (2007).

[13] Y. V. Kovchegov and H. Weigert, Triumvirate of running couplings in small-x evolution, Nucl. Phys. A784, 188 (2007).

[14] I. Balitsky and G. A. Chirilli, Next-to-leading order evolution of color dipoles, Phys. Rev. D 77, 014019 (2008).

[15] I. Balitsky and G. A. Chirilli, Rapidity evolution of Wilson lines at the next-to-leading order, Phys. Rev. D 88, 111501 (2013).

[16] A. Kovner, M. Lublinsky, and Y. Mulian, Jalilian-Marian, Iancu, McLerran, Weigert, Leonidov, Kovner evolution at next to leading order, Phys. Rev. D 89, 061704 (2014).

[17] A. Kovner, M. Lublinsky, and Y. Mulian, NLO JIMWLK evolution unabridged, J. High Energy Phys. 08 (2014) 114. 
[18] M. Lublinsky and Y. Mulian, High energy QCD at NLO: From light-cone wave function to JIMWLK evolution, J. High Energy Phys. 05 (2017) 097.

[19] Y. V. Kovchegov and E. Levin, Quantum Choromodynamics at High Energies, Cambridge Monographs on Particle Physics, Nuclear Physics and Cosmology (Cambridge University Press, Cambridge, England, 2012).

[20] L. V. Gribov, E. M. Levin, and M. G. Ryskin, Semihard processes in QCD, Phys. Rep. 100, 1 (1983).

[21] A.H. Mueller and J. Qiu, Gluon recombination and shadowing at small values of $x$, Nucl. Phys. B268, 427 (1986).

[22] L. McLerran and R. Venugopalan, Computing quark and gluon distribution functions for very large nuclei, Phys. Rev. D 49, 2233 (1994); Gluon distribution functions for very large nuclei at small transverse momentum, Phys. Rev. D 49, 3352 (1994); Green?s function in the color field of a large nucleus, Phys. Rev. D 50, 2225 (1994); Fock space distributions, structure functions, higher twists, and small $x$, Phys. Rev. D 59, 094002 (1999).

[23] J. Jalilian-Marian, A. Kovner, A. Leonidov, and H. Weigert, The BFKL equation from the Wilson renormalization group, Nucl. Phys. B504, 415 (1997).

[24] J. Jalilian-Marian, A. Kovner, A. Leonidov, and H. Weigert, The Wilson renormalization group for low $\mathrm{x}$ physics: Towards the high density regime, Phys. Rev. D 59, 014014 (1998).

[25] A. Kovner, J. G. Milhano, and H. Weigert, Relating different approaches to nonlinear QCD evolution at finite gluon density, Phys. Rev. D 62, 114005 (2000).

[26] E. Iancu, A. Leonidov, and L. D. McLerran, Nonlinear gluon evolution in the color glass condensate. I, Nucl. Phys. A692, 583 (2001).

[27] E. Iancu, A. Leonidov, and L. D. McLerran, The renormalization group equation for the color glass condensate, Phys. Lett. B 510, 133 (2001).

[28] E. Ferreiro, E. Iancu, A. Leonidov, and L. McLerran, Nonlinear gluon evolution in the color glass condensate. II, Nucl. Phys. A703, 489 (2002).

[29] J. L. Albacete, N. Armesto, J. G. Milhano, P. Quiroga-Arias, and C. A. Salgado, AAMQS: A non-linear QCD analysis of new HERA data at small-x including heavy quarks, Eur. Phys. J. C 71, 1705 (2011).

[30] K. Kutak and S. Sapeta, Gluon saturation in dijet production in $\mathrm{p}-\mathrm{Pb}$ collisions at Large Hadron Collider, Phys. Rev. D 86, 094043 (2012).

[31] T. Lappi and H. Mäntysaari, Direct numerical solution of the coordinate space Balitsky-Kovchegov equation at next to leading order, Phys. Rev. D 91, 074016 (2015).

[32] E. Iancu, J. D. Madrigal, A. H. Mueller, G. Soyez, and D. N. Triantafyllopoulos, Collinearly-improved BK evolution meets the HERA data, Phys. Lett. B 750, 643 (2015).

[33] B. Ducloué, H. Hänninen, T. Lappi, and Y. Zhu, Deep inelastic scattering in the dipole picture at next-to-leading order, Phys. Rev. D 96, 094017 (2017).

[34] G. Beuf, H. Hänninen, T. Lappi, and H. Mäntysaari, Color glass condensate at next-to-leading order meets HERA data, Phys. Rev. D 102, 074028 (2020).

[35] A. Kovner and U. A. Wiedemann, Nonlinear QCD evolution: Saturation without unitarization, Phys. Rev. D 66, 051502 (2002).
[36] A. Kovner and U. A. Wiedemann, Perturbative saturation and the soft Pomeron, Phys. Rev. D 66, 034031 (2002);

[37] A. Kovner and U. A. Wiedemann, No froissart bound from gluon saturation, Phys. Lett. B 551, 311 (2003).

[38] E. Ferreiro, E. Iancu, K. Itakura, and L. McLerran, Froissart bound from gluon saturation, Nucl. Phys. A710, 373 (2002).

[39] M. Froissart, Asymptotic behavior and subtractions in the mandelstam representation, Phys. Rev. 123, 1053 (1961); A. Martin, Scattering Theory: Unitarity, Analitycity and Crossing, Lecture Notes in Physics (Springer-Verlag, BerlinHeidelberg-New-York, 1969).

[40] J. Berger and A. M. Stasto, Small x nonlinear evolution with impact parameter and the structure function data, Phys. Rev. D 84, 094022 (2011).

[41] J. Berger and A. Stasto, Numerical solution of the nonlinear evolution equation at small $\mathrm{x}$ with impact parameter and beyond the LL approximation, Phys. Rev. D 83, 034015 (2011).

[42] J. Cepila, J. G. Contreras, and M. Matas, Collinearly improved kernel suppresses Coulomb tails in the impactparameter dependent Balitsky-Kovchegov evolution, Phys. Rev. D 99, 051502 (2019).

[43] D. Bendova, J. Cepila, J. G. Contreras, and M. Matas, Solution to the Balitsky-Kovchegov equation with the collinearly improved kernel including impact-parameter dependence, Phys. Rev. D 100, 054015 (2019).

[44] C. Contreras, E. Levin, and R. Meneses, BFKL equation in the next-to-leading order: solution at large impact parameters, Eur. Phys. J. C 79, 842 (2019).

[45] G. P. Lepage and S. J. Brodsky, Exclusive Processes in Quantum Chromodynamics: The Form-Factors of Baryons at Large Momentum Transfer, Phys. Rev. Lett. 43, 545 (1979); 43, 1625(E) (1979).

[46] S. Bondarenko, M. Kozlov, and E. Levin, QCD saturation in the semi-classical approach, Nucl. Phys. A727, 139 (2003).

[47] K. J. Golec-Biernat and M. Wusthoff, Saturation in diffractive deep inelastic scattering, Phys. Rev. D 60, 114023 (1999); Saturation effects in deep inelastic scattering at low $Q^{2}$ and its implications on diffraction, Phys. Rev. D 59, 014017 (1998).

[48] J. Bartels, K. J. Golec-Biernat, and H. Kowalski, A modification of the saturation model: DGLAP evolution, Phys. Rev. D 66, 014001 (2002).

[49] H. Kowalski and D. Teaney, An impact parameter dipole saturation model, Phys. Rev. D 68, 114005 (2003).

[50] E. Iancu, K. Itakura, and S. Munier, Saturation and BFKL dynamics in the HERA data at small x, Phys. Lett. B 590, 199 (2004).

[51] H. Kowalski, L. Motyka, and G. Watt, Exclusive diffractive processes at HERA within the dipole picture, Phys. Rev. D 74, 074016 (2006).

[52] H. Kowalski, T. Lappi, and R. Venugopalan, Nuclear Enhancement of Universal Dynamics of High Parton Densities, Phys. Rev. Lett. 100, 022303 (2008).

[53] H. Kowalski, T. Lappi, C. Marquet, and R. Venugopalan, Nuclear enhancement and suppression of diffractive structure functions at high energies, Phys. Rev. C 78, 045201 (2008).

[54] G. Watt and H. Kowalski, Impact parameter dependent colour glass condensate dipole model, Phys. Rev. D 78, 014016 (2008). 
[55] E. Levin and A. H. Rezaeian, Gluon saturation and inclusive hadron production at LHC, Phys. Rev. D 82, 014022 (2010).

[56] A. H. Rezaeian, CGC predictions for $\mathrm{p}+\mathrm{A}$ collisions at the LHC and signature of QCD saturation, Phys. Lett. B 718, 1058 (2013).

[57] E. Levin and A. H. Rezaeian, Gluon saturation and energy dependence of hadron multiplicity in pp and AA collisions at the LHC, Phys. Rev. D 83, 114001 (2011).

[58] E. Levin and A. H. Rezaeian, Hadron multiplicity in pp and AA collisions at LHC from the color glass condensate, Phys. Rev. D 82, 054003 (2010).

[59] D. Boer, M. Diehl, R. Milner, R. Venugopalan, W. Vogelsang, D. Kaplan, H. Montgomery, S. Vigdor et al., Gluons and the quark sea at high energies: Distributions, polarization, tomography, arXiv:1108.1713.

[60] T. Lappi and H. Mantysaari, Incoherent diffractive J/Psiproduction in high energy nuclear DIS, Phys. Rev. C 83, 065202 (2011).

[61] T. Toll and T. Ullrich, Exclusive diffractive processes in electron-ion collisions, Phys. Rev. C 87, 024913 (2013).

[62] P. Tribedy and R. Venugopalan, Saturation models of HERA DIS data and inclusive hadron distributions in $\mathrm{p}+\mathrm{p}$ collisions at the LHC, Nucl. Phys. A850, 136 (2011); A859, 185(E) (2011).

[63] P. Tribedy and R. Venugopalan, QCD saturation at the LHC: Comparisons of models to $\mathrm{p}+\mathrm{p}$ and $\mathrm{A}+\mathrm{A}$ data and predictions for $\mathrm{p}+\mathrm{Pb}$ collisions, Phys. Lett. B 710, 125 (2012); 718, 1154(E) (2013).

[64] A. H. Rezaeian, M. Siddikov, M. Van de Klundert, and R. Venugopalan, IP-Sat: Impact-Parameter dependent saturation model revised, Proc. Sci., DIS2013 (2013) 060 [arXiv:1307.0165]; Analysis of combined HERA data in the impact-parameter dependent saturation model, Phys. Rev. D 87, 034002 (2013).

[65] A. H. Rezaeian and I. Schmidt, Impact-parameter dependent color glass condensate dipole model and new combined HERA data, Phys. Rev. D 88, 074016 (2013).

[66] C. Contreras, E. Levin, and I. Potashnikova, CGC/saturation approach: A new impact-parameter dependent model, Nucl. Phys. A948, 1 (2016).

[67] E. M. Levin and M. G. Ryskin, High-energy hadron collisions in QCD, Phys. Rep. 189, 268 (1990).

[68] E. M. Levin and M. G. Ryskin, The shrinkage of the diffraction peak of the bare Pomeron in QCD, Sov. J. Nucl. Phys. 50, 881 (1989); Yad. Fiz. 50, 1417 (1989) [Z. Phys. C 48, 231 (1990)].

[69] E. Levin and C. I. Tan, Heterotic Pomeron: A unified treatment of high-energy hadronic collisions in QCD, in Santiago de Compostela 1992, Proceedings, Multiparticle dynamics, 568-575 and Fermilab Batavia - FERMILABConf-92-391 (92/09,rec.Jan.93) p. 9 (303600) Brown University Providence - BROWN-HET-889; arXiv:hep-ph/ 9302308.

[70] D. Y. Ivanov, R. Kirschner, E. M. Levin, L. N. Lipatov, L. Szymanowski, and M. Wusthoff, The BFKL Pomeron in (2+ 1)-dimensional QCD, Phys. Rev. D 58, 074010 (1998).

[71] D. Kharzeev and E. Levin, Scale anomaly and "soft" Pomeron in QCD, Nucl. Phys. B578, 351 (2000).
[72] D. E. Kharzeev, Y. V. Kovchegov, and E. Levin, QCD instantons and the soft Pomeron, Nucl. Phys. A690, 621 (2001).

[73] S. Bondarenko, E. Levin, and C. I. Tan, High energy amplitude as an admixture of "soft" and "hard" Pomerons, Nucl. Phys. A732, 73 (2004).

[74] E. Levin, Large $\boldsymbol{b}$ behavior in the CGC/saturation approach: BFKL equation with pion loops, Phys. Rev. D 91, 054007 (2015).

[75] O. V. Kancheli, On the parton picture of Froissart asymptotic behavior, arXiv:1609.07657.

[76] E. Gotsman and E. Levin, Large impact parameter behavior in the CGC/saturation approach: A new nonlinear equation, Phys. Rev. D 101, 014023 (2020).

[77] V. N. Gribov, Strong Interactions of Hadrons at High Energies (Cambridge University Press, Cambridge, England, 2008); The Theory of Complex Angular Momenta: Gribov Lectures on Theoretical Physics, Cambridge Monographs on Mathematical Physics (Cambridge University Press, Cambridge, England, 2003), p. 312; Space-time description of hadron interactions at high-energies, arXiv: hep-ph/0006158; Inelastic processes at super high-energies and the problem of nuclear cross-sections, Yad. Fiz. 9, 640 (1969) [Sov. J. Nucl. Phys. 9, 369 (1969)].

[78] V. A. Khoze, A. D. Martin, M. G. Ryskin, and W. J. Stirling, The spread of the gluon $k_{t}$-distribution and the determination of the saturation scale at hadron colliders in resummed NLL BFKL, Phys. Rev. D 70, 074013 (2004).

[79] V.S. Fadin and L. N. Lipatov, BFKL Pomeron in the next-to-leading approximation, Phys. Lett. B 429, 127 (1998).

[80] M. Ciafaloni and G. Camici, Energy scale(s) and next-toleading BFKL equation, Phys. Lett. B 430, 349 (1998).

[81] A.S. Vera, An 'All-poles' approximation to collinear resummations in the Regge limit of perturbative QCD, Nucl. Phys. B722, 65 (2005).

[82] A. H. Mueller and D. N. Triantafyllopoulos, The energy dependence of the saturation momentum, Nucl. Phys. B640, 331 (2002).

[83] A. D. Polyanin and V. F. Zaitsev, Handbook of Nonlinear Partial Differential Equations (Chapman and Hall/CRC Press, Raca Baton, New York, London, Tokyo, 2004).

[84] E. Gotsman, E. Levin, U. Maor, and E. Naftali, The components of the $\gamma * \gamma *$ cross-section, Eur. Phys. J. C 14, 511 (2000).

[85] E. Iancu, K. Itakura, and L. McLerran, Geometric scaling above the saturation scale, Nucl. Phys. A708, 327 (2002).

[86] E. Levin, Dipole-dipole scattering in CGC/saturation approach at high energy: Summing Pomeron loops, J. High Energy Phys. 11 (2013) 039.

[87] F. D. Aaron et al. (H1 and ZEUS Collaborations), Combined measurement and QCD analysis of the inclusive ep scattering cross sections at HERA, J. High Energy Phys. 01 (2010) 109.

[88] H. Abramowicz et al. (H1 and ZEUS Collaborations), Combination and QCD analysis of charm production cross section measurements in deep-inelastic ep scattering at HERA, Eur. Phys. J. C 73, 2311 (2013). 
[89] V. Andreev et al. (H1 Collaboration), Measurement of inclusive $e p$ cross sections at high $Q^{2}$ at $\sqrt{s}=225$ and $252 \mathrm{GeV}$ and of the longitudinal proton structure function $F_{L}$ at HERA, Eur. Phys. J. C 74, 2814 (2014); F. D. Aaron et al. (H1 Collaboration), Measurement of the proton structure function $F_{L}\left(x, Q^{2}\right)$ at low $x$, Phys. Lett. B 665, 139 (2008).

[90] H. Abramowicz et al. (ZEUS Collaboration), Deep inelastic cross-section measurements at large $y$ with the ZEUS detector at HERA, Phys. Rev. D 90, 072002 (2014); S. Chekanov et al. (ZEUS Collaboration), Measurement of the longitudinal proton structure function at HERA, Phys. Lett. B 682, 8 (2009).
[91] D. E. Kharzeev, The mass radius of the proton, Phys. Rev. D 104, 054015 (2021).

[92] K. A. Mamo and I. Zahed, Nucleon mass radii and distribution: Holographic QCD, lattice QCD and GlueX data, Phys. Rev. D 103, 094010 (2021).

[93] A. Dumitru, D. E. Kharzeev, E. M. Levin, and Y. Nara, Gluon saturation in $p A$ collisions at the LHC: KLN model predictions for hadron multiplicities, Phys. Rev. C 85, 044920 (2012).

[94] C. Contreras, E. Levin, and M. Sanhueza, Soft Pomeron in the Colour Glass Condensate approach (to be published). 